Proceedings of the Pakistan Academy of Sciences:

A: Physical and Computational Sciences 58(3): 15-29 (2021)

Copyright (C) Pakistan Academy of Sciences

ISSN (Print): 2518-4245; ISSN (Online): 2518-4253

https://doi.org/10.53560/PPASA(58-3)602

\title{
Analytic Preview of Spectral Contribution of the Neutrinos Emission from Deep Space during Specified Period
}

\author{
Syed Muhammad Ali Abbas Naqvi', Faisal Ahmed Khan Afridi ${ }^{1}$, Bulbul Jan ${ }^{2 *}$, \\ Muhammad Ayub Khan Yousuf Zai ${ }^{1,3}$, Mirza Jawad Baig ${ }^{1}$, Arshad Hussain ${ }^{3}$, \\ Syed Muhammad Haroon Rashid ${ }^{1}$, and Abid Hussain ${ }^{3}$.
}

\author{
${ }^{1}$ Institute of Space Science and Technology, University of Karachi, Pakistan \\ ${ }^{2}$ Department of Basic Sciences, Dawood University of Engineering and Technology \\ Karachi, Pakistan \\ ${ }^{3}$ Department of Applied Physics, University of Karachi, Pakistan.
}

\begin{abstract}
The earlier period investigations of Neutrinos emission from deep space indicate the existence of this particle which has been detected in various laboratories of the world. These laboratories are responsible for recording neutrinos emission originated from deep space. One laboratory situated near Sudbury, Ontario, Canada known as Sudbury Neutrino Observatory (SNO). Another laboratory is in Japan known as Kamikanado. We obtained a set of observations from both labs. It is obvious that the observations recorded at SNO have been utilized in this manuscript. From the same communication, the neutrino behaviour could be better understood by analyzing the SNO observed data set-I from November 1999 to May 2001 of $\mathrm{D}_{2} \mathrm{O}$, while dataset-II is from July 2001 to August 2003 of salt water with particulars recorded, Run start Time since midnight. The samples used in this paper are 250 entries from both the observations sets. In this presentation, the most spectacular exhibition of the neutrino flux in its frequency components of the particles have been depicted in the form of a Periodogram that identifies covariance structure in the neutrino flux reaching this biosphere and parametric values obtained in this paper have been tabulated in various table frame of work. This communication does claim the neutrino emission characteristics in the real world. It has also been known from the literature survey that the same kind of work has not been framed in the third world countries where no neutrino detection laboratories exist. This piece of information will be beneficial for the private and public organizations where the experts are trying to explore deep space emissions detection and their characterization like the present study.
\end{abstract}

Keywords: Neutrino Flux, Deep Space, SNO, Periodogram Manifestation of Spectral Contribution.

\section{INTRODUCTION}

It has been known for a long time that the basic structure of an atom, according to Rutherford comprises a nucleus having proton and neutron at the centre of the atom and electrons moving around the nucleus. It has been explored that during the 19th century, the origin of neutrino was linked to the discovery of $\beta$ decay of nuclei. At the same time after Solvay conference the famous Physicist Fermi established theory of beta decay and developed the structure of beta spectrum and the interpretation of rest mass of the neutrino [1]. Pauli during the year 1930 affirmed the familiarity of energy and momentum conservation of the process of $\beta$-decays via hypothesizing neutrino [2, 3, and 4].

Neutrinos are known as neutral leptons that are not well measured since long and detection of neutrinos is difficult as neutrinos rarely interact with other matter. Frederick Reines, Clyde Cowan, $\mathrm{Jr}$, and collaborators experimentally observed neutrino interacting with matter first time at the Savannah River Plant in South Carolina in 1956.

Received: December 2020; Accepted: July 2021

*Corresponding Author: Bulbul Jan <bulbul@duet.edu.pk> 
The source for their neutrino was a nuclear reactor (i.e. production of antineutrinos from beta decay). Sudbury Neutrino detector at Ontario started collecting data in Oct 1999. Super Kamiokande neutrino detector Japan became operational in April 1996.

We have found that neutrino laboratories in the world are detecting neutrinos coming to earth from deep space. Solar neutrino research entered a new era in April 1996, when the Super-Kamiokande experiment began to operate. In the previous era, solar neutrinos were detected by four outstanding experiments; (a) the radiochemical Homes take chlorine experiment, (b) the Kamiokande water, Cherenkov experiment, and the two (c and d) radiochemical gallium experiments, GALLEX and SAGE. In these four exploratory experiments, typically less than or of the order of 50 neutrino events were observed per year $[5,6,7,8,9,10,11$, 12].

It has been found that the neutrino investigation has played a pivotal role for about four decades to study and understand the existence of neutrino from the evolution of the Sun, extragalactic supernovae and cosmic rays [13]. Moreover, the extra variability of solar neutrino flux has been studied since the work done in the Home stake experiment, where it showed the existence of neutrino from the Sun. This has been suggested by Sheldon in an early attempt that the neutrino flux depends on the periods of solar activities. The work done represented the temporal behaviour of Sunspot dynamics during the 11 years solar cycle [14, 15, and 16].

It has also been observed that instead of temporal characteristic, the spatial scenario of solar activities related to the solar equator for the 11-year cycle, it can be displayed by the famous "butterfly diagram" that included both time and space domains characteristics. With these large scale domains, the existence of short duration stochastic variability is formulated [17]. Incorporation of Homestake set of observations as revealed by Sakuraiwho depicted the existence of periodicity during quasi-biennial for both solar neutrino flux and sunspot numbers $[10,18]$.

Results of Sudbury Neutrino Observatory addressed the Solar Neutrino Problem, suggested neutrino oscillation from electron neutrino to other flavours on the way to earth from Sun. Data recorded at Sudbury is consistent with the expected neutrino flux of solar models assuming "neutrino oscillation" is the reason for observation of neutrinos other than electron neutrinos.

The observation vessel at SNO is surrounded by roughly 9,500 PMTs of $20-\mathrm{cm}$ dia, sensitive to Cherenkov radiation, the radiation is produced by muon neutrino and electron neutrino events as shown in Fig.1a. SNO data records the start time of Cherenkov radiation (neutrino interaction) observed after midnight and the duration of that Cherenkov radiation (length of the interaction/reaction).

In this communication, we intend to examine the spectral contributions of the neutrino emissions from deep space during specified periods. Neutrinos we known as being leptons, are of three kinds called the electron neutrino, muon neutrino and the tau neutrino $\left(v_{-} \mathrm{e}, v_{-} \mu, v_{-} \tau\right)$. The Neutrino detector station at Sudbury, Ontario, Canada called "Sudbury Neutrino Observatory (SNO)" where the detection of this particle is taken place and the collected samples of set of observations will be analyzed here in Pakistan by our research group. Neutrino flux will be assessed using mathematical Statistical approaches.

\subsection{The Manifestations of Neutrino emissions from different processes}

During the start of 20th century, Wolfgang Pauli proposed the innocent particle in Universe, known as neutrino as energy distribution of electrons in beta decays were found continuous After few years Enrico Fermi in theoretical formalism for the $\beta$-decay included Pauli's new particle. Neutrino, which is considered as electrically neutral and possessing formidable mass, therefore its detection were challenged in experimental laboratories to verify its character [4].

It has been found that solar neutrino experiments were originally conceived as a way to demonstrate that nuclear reactions are responsible for energy generation in stars. When solar neutrinos were first detected, the measured flux was much less than what solar models predicted [19]. The famous reactions related to these emissions are illustrated 


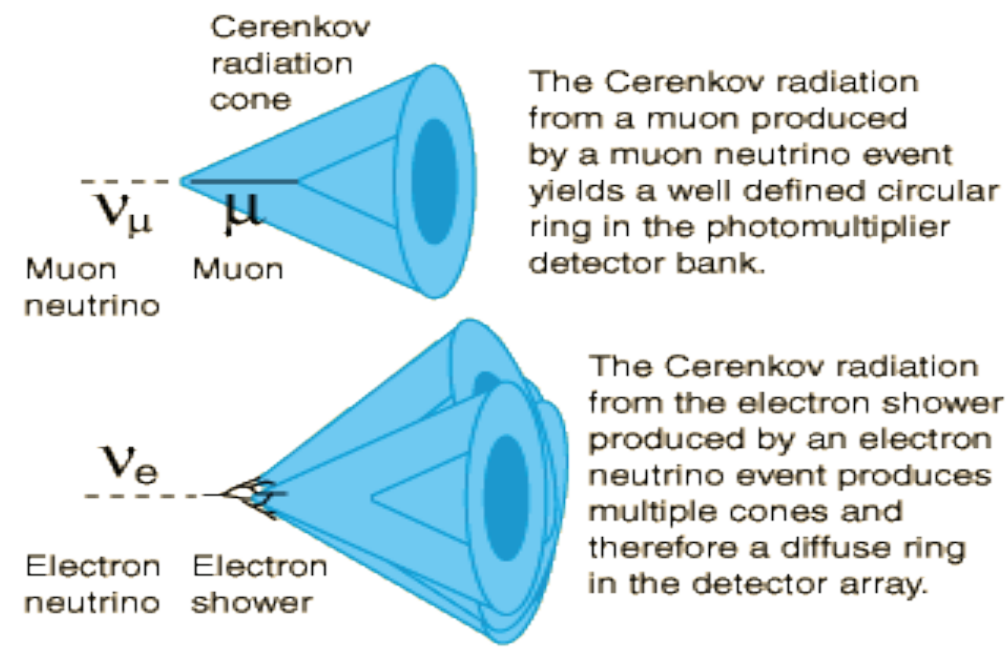

Fig. 1a. Depiction of cherenkov radiation, produced by muon neutrino and electron neutrino events.

as follows:

a. The pp chain of reactions is the dominant process in stars like our Sun. Whereas the CNO cycle becomes more significant for stars that are heavier.

b. Important emissions from deep space will be considered as solar neutrinos that include the following nuclear reactions:

$$
\begin{gathered}
p+p \rightarrow d+e^{+}+v_{e} \\
p+e^{-}+p \rightarrow d+v_{e} \\
{ }^{7} B e+e^{-} \rightarrow{ }^{7} L i+v_{e} \\
{ }^{8} B \rightarrow 2 \alpha+e^{+}+v_{e}
\end{gathered}
$$

It has been depicted that emitted neutrinos in various chains of the above reactions have characteristic energies [20]. One of the chain reactions is responsible for hydrogenation leading to a helium network. It gives a reaction sequence that consequently produces the following reactions:

$$
4{ }_{1}^{1} H \rightarrow{ }_{2}^{4} \mathrm{He}+2 e^{+}+2 v_{e}+2 \gamma
$$

The second preview of the chemical reactions indicates creation of helium-4 nucleus from Hydrohen-1. For this purpose we are in a position to introduce carbon-Nitrogen-Oxygen (CNO) cycle, nitrogen and oxygen are used as catalyst and then regenerated during reaction. According to this cycle the following reactions are generated:

$$
\begin{aligned}
& { }_{6}^{12} \mathrm{C}+{ }_{1}^{1} H \rightarrow{ }_{7}^{13} \mathrm{~N}+\gamma \\
& { }_{6}^{13} \mathrm{C}+{ }_{1}^{1} H \rightarrow{ }_{7}^{14} \mathrm{~N}+\gamma \\
& { }_{7}^{14} \mathrm{~N}+{ }_{1}^{1} H \rightarrow{ }_{8}^{15} \mathrm{O}+\gamma
\end{aligned}
$$

$$
{ }_{7}^{15} \mathrm{~N}+{ }_{1}^{1} \mathrm{H} \rightarrow{ }_{6}^{12} \mathrm{C}+{ }_{2}^{4} \mathrm{He}
$$

In the above reactions, the neutrino flux has been indicated with the formation of helium nucleus [21].

From the above, the existence of neutrino from different perspectives is obvious. Therefore we intended to study the analytic preview of spectral contributions of the neutrino emission from deep space during the specified period.

\section{SETS OF OBSERVATIONS AND METHODS OF INCORPORATION}

\subsection{Source of Observations}

The Sudbury Neutrino Observatory recorded two sets of observations, first starting from $2^{\text {nd }}$. Nov 1999 to $27^{\text {th }}$ May 2001 using heavy water $\left(\mathrm{D}_{2} \mathrm{O}\right)$ as an interacting medium, having parameters recorded variables, Run start Time since midnight and Length of run, in Sec, having 559 entries and second set of observations starting from $26^{\text {th }}$ July 2001 to $28^{\text {th }}$ Aug 2003 using salt water as a medium of interactions with same particulars recorded, having 1212 entries are taken.

It is noted that for proper symmetric comparison and analysis, samples are so taken that coincides for solar year time starting from $2^{\text {nd }}$ Nov 1999 for heavy water $\left(\mathrm{D}_{2} \mathrm{O}\right)$ and $2^{\text {nd }}$ Nov 2001 for salt water set of observations thereby celestial position of our Planet Earth that is orbiting round the Sun in the Galaxy should be as same as possible at the time of 
recording of observations.

It has been known that solar neutrinos have been detected at the Sudbury Neutrino Observatory (SNO) showing the decay of $8 \mathrm{~B}$ via the charged current (CC) reaction on deuterium and elastic scattering (ES) of electrons. This CC reaction seemed to be more sensitive in case of $v_{\mathrm{e}}$ 's and the ES reaction has the sensitivity to $v_{\mu}{ }^{\prime} s$ and $v_{\tau}{ }_{\tau} s$ [22].

The report on the measurement of the total flux of active $8 \mathrm{~B}$ neutrino at $\mathrm{SNO}$ from the solar emission indicates neutrino flavor transformation. The CC and ES reaction rates are consistent with earlier results and with the NC reaction rate under the hypothesis of flavour transformation. It has been found that the $8 \mathrm{~B}$ neutrino flux is in the agreement of Standard solar model. The abrupt changes on a daily basis have been reported in the $\mathrm{CC}, \mathrm{NC}$, and ES at SNO with different reaction rates. These results showed the direct measurement of the day and night asymmetries in the neutrinos A Global fit to SNO's day and night energy spectra and set of observations from other solar neutrino experiments strongly favours the large mixing angle (LMA) neutrino oscillation analysis [23].

It has been investigated that at SNO the total flux of active8B neutrinos has been precisely measured from the Sun with energy dependence of the electron neutrino with their survival probability. According to the experimental result this flux has equivalency with the calculated solar model [24].

All three fluxes (CC, ES and NC) data from the pure heavy water $\mathrm{D}_{2} \mathrm{O}$ phase presented and analyzed, were in general derived under the assumption of an energy-independent survival probability of undistorted $8 \mathrm{~B}$ energy spectrum. The determination of results at SNO for salt fluxes also showed energy dependence and survival probability of same type of neutrinov_e. SNO Collaboration based on correlations between salt results and its heavy water results, comments "there are correlations being the same detector, these are not large, since some changes have been made to both the detector" [24]. The heavy water target in SNO gives three types of reactions for investigating solar neutrinos. Thus the SNO can provide substantial intuition for the nature of neutrinos indicating model for Solar Standard $[25,26]$.

\subsection{Sudbury Neutrino Observatory (SNO) in Canada}

In this section, the Sudbury Neutrino Observatory (SNO) described its internal structure about the Cerenkov detector located $2 \mathrm{~km}$ underground in INCO's Creighton Mine, near Sudbury, Ontario, Canada. There is a1000 tons heavy water $\left(\mathrm{D}_{2} \mathrm{O}\right)$ located with the geographical position the observatory mentioned as (Latitude: $46^{\circ} 27^{\prime}$ 59.99" N, Longitude: $\left.-81^{\circ} 10^{\prime} 13.20^{\prime \prime} \mathrm{W}\right)$. The underground laboratory has been enlarged and continues to operate other experiments at SNOLAB $[23,26,27,28,29,30,31]$.

\subsection{Mode of Computation of parametric values for neutrino flux}

In this section, parametric values of the neutrino emission can be computed indicating time domain and frequency-domain concepts. These domains illustrate the spectral and Periodogram analyses.

Time Series: $\mathrm{Xt}=A \operatorname{Cos}(2 \pi \omega t+\phi)$

Useful trigonometric identity:

$A \operatorname{Cos}(2 \pi \omega t+\phi)=\beta_{1} \operatorname{Cos}(2 \pi \omega t)+\beta_{2} \operatorname{Sin}(2 \pi \omega t)$

Where $\beta_{1}=\mathrm{A} \operatorname{Cos}(\phi)$ and $\beta_{2}=-\mathrm{A} \operatorname{Sin}(\phi)$. This identity is used when we determine the Periodogram of a set of observations.

It has been examined that Spectral and periodogram diagnostics are related to energies and frequencies contained in the spectrum. In the case of spectral analyses, the entire sets of observations have been decomposed into its sine wave components. As regards the decomposition of a time-series into a sum of weighted sinusoidal functions is to utilize Fourier transform comprising discrete and continuous expressions. Similarly, a periodogram fascination is considered as an estimate of signal spectral density. Sophistication of Periodogram is obvious if we plot amplitude vs frequency characteristics of finite impulse response (FIR) filters and window functions. It has been indicated that the Fast Fourier Transform (FFT) spectrum analyzers are also utilized as a periodograms describing the time sequence. From the knowledge of spectral analyses it has been 
examined that the power spectral density of a continuous function, is the Fourier transform of its auto-correlation function.

$F\{x(t) * x(-t)\}=X(f) X^{*}(f)=|X(f)|^{2}$

It has been pointed out that a time series can be expressed as a combination of cosine (or sine) waves with differing periods and amplitudes. The periedogram can be expressed as a line spectrum that does display an observed time series as superposition of sinusoidal waves with various frequencies as shown in the following relationship:

$$
X_{t}=\alpha \operatorname{Cos}(\omega t)+\beta \operatorname{Sin}(\omega t)+Z_{t}, \quad(\mathrm{t}=1-\mathrm{n})
$$

Where $\left\{Z_{t}\right\}$ is white noise sequence and it is normally and identically distributed, $\omega=2 \pi / p$ is the frequency of the cyclic fluctuations and $\alpha$ and $\beta$ are parameters that are estimated by least squares. It has been stated that a Periadogram also does helps in recognizing applicable Auto Regressive model.

An examination of autocorrelation, partial autocorrelation function and line spectrum related to a time series also helps us in the detection of the underlying auto regression model. It is said that if the autocorrelation function is exponentially decaying with one significant partial autocorrelation and line spectrum support (Low frequencies if the AR coefficient $\beta_{1}$ is positive, higher frequencies if $\beta_{1}$ is negative), then the under lying model is an AR (1) model.

It has been observed that $95 \%$ confidence interval is used to estimate the autocorrelation function useful for displaying the spectrum of the neutrino flux reaching the biosphere $[32,33,34,35$, $36,37]$.

Moreover, the spectral density $S(\omega)$ of a stationary random process is the Fourier transform of the autocorrelation function $\mathrm{R}(\tau)$. It is given by:

$$
S(\omega)=\frac{1}{2 \pi} \int_{-\infty}^{\infty} R(\tau) e^{-j w T} d w
$$

The inverse, which also holds true, is

$$
R(\tau)=\frac{1}{2 \pi} \int_{-\infty}^{\infty} S(\omega) e^{-j w T} d w
$$

If $\tau=0$

$$
R(0)=\int_{-\infty}^{\infty} S(\omega) d w=E\left[x^{2}\right]
$$

That is, the mean square value of a stationary random process $x$ is the area under the $S(\omega)$ against frequency curve. If the spectral density of a process has a significant value over a narrower range of frequencies, but one that is nevertheless still wide compared with the centre frequency of the band, it is termed a wide-band process. If the frequency range is narrow compared with the centre frequency it is termed a narrow-band process. These processes frequently occur in engineering practices because real systems respond strongly and act as a filter. Power spectral density function (PSDF) illustrates the strength of the variations (energy) as a function of frequency. Computation of PSDF is done directly by the method called Fast Fourier Transform.

\section{RESULTS AND DISCUSSIONS}

In this section, we have tried to check the nature of our set of observations that we obtained from SNO. For this purpose we perform a Stationary test to assess the non stationary and stationary nature (set of observations is stationary or non-stationary). Sets of observations I and II, variable (Run start Time since midnight) starting from 02 Nov 1999 using heavy water $\left(\mathrm{D}_{2} \mathrm{O}\right)$ and 2 nd of Nov 2001 using salt water is done, the statistical parameters recorded are tabulated in Table 1.

For Set of observations I, the ADF test value $\tau=-16.97750$, which is more negative than even the $1 \%$ significance level $(-3.456514)$, and value of " $p$ " is 0.000 which less than the significance level of 0.05 as shown in Table 1 , so we reject the null hypothesis of unit root test, in other words, the run start time since midnight of $\mathrm{D}_{2} \mathrm{O}$ set of observations I, is a stationary series. Similarly, for set of observations II, the ADF test value $\tau=-13.69809$, which is more negative than even the $1 \%$ significance level $(-3.456514)$, and value of " $p$ " is 0.000 which less than the significance level of 0.05 as shown in the Table 1 , so we can reject the null of non-stationary data, in other words the run start time since midnight of salt water set of observations II, is a stationary series. AIC, SC and coefficient of determination $\left(\mathrm{R}^{2}\right)$ results for the trend detection for both set of observations I \& II are tabulated in Table $1 \mathrm{~b}$. 
Table 1. Stationary test for SNO set of observations I \& II, variable (Run start Time since midnight) of heavy water $\left(\mathrm{D}_{2} \mathrm{O}\right)$ and Salt water parametric values are recorded

\begin{tabular}{|c|c|c|c|c|c|}
\hline & \multicolumn{2}{|c|}{$\mathrm{D}_{2} \mathrm{O}$ Heavy Water } & \multicolumn{2}{|c|}{ Salt Water } \\
\hline & & $\mathrm{T}-$ Statistic & Probability & $\mathrm{T}-$ Statistic & Probability \\
\hline \multicolumn{2}{|c|}{ Augmented Dicky-Fuller test Statistic } & -16.97750 & $\mathrm{P}<0.001$ & -13.69809 & 0.0000 \\
\hline \multirow{3}{*}{$\begin{array}{l}\text { Test critical } \\
\text { values }\end{array}$} & $1 \%$ Level & -3.456514 & & -3.456514 & \\
\hline & $5 \%$ Level & -2.872950 & & -2.872950 & \\
\hline & $10 \%$ Level & -2.572925 & & -2.572925 & \\
\hline \multicolumn{2}{|l|}{ Trend Line } & $y=-1.08 x$ & 9655.17 & $y=-0.86 x+3$ & 4.42 \\
\hline
\end{tabular}

Table 2. Largestperiodogram peaks of Time of start of run since midnight of $\mathrm{SNO}_{2} \mathrm{O}$ set of observations

\begin{tabular}{|c|c|c|c|c|c|}
\hline \multirow[t]{2}{*}{ Variable } & \multicolumn{5}{|c|}{ Run start time since Midnight - Five largest value } \\
\hline & (1) & (2) & (3) & (4) & (5) \\
\hline Periodogram peaks & $4.56 \times 10^{9}$ & $3.72 \times 10^{9}$ & $3.64 \times 10^{9}$ & $3.58 \times 10^{9}$ & $3.47 \times 10^{9}$ \\
\hline Frequency & 0.4880 & 0.1640 & 0.3840 & 0.2880 & 0.3360 \\
\hline Period & 2.0492 & 6.0976 & 2.6042 & 3.4722 & 2.9762 \\
\hline
\end{tabular}

3.1 Categorical aspects of Periodogram for the Time to start the run (Neutrino interaction) since midnight of SNO set of observations using $\mathrm{D}_{2} \mathrm{O}$ and salt water:

The given sketch of the set observations meaningfully indicates the magnitude of Periodogram vs. frequency of run in our 1st set, heavy water $\left(\mathrm{D}_{2} \mathrm{O}\right)$ that is represented in Figure 1. Five major peak values of Time of start of run since midnight of sample, having 250 entries from $2^{\text {nd }}$ Nov 1999 to $24^{\text {th }}$ Jun 2000 listed in the Table 2. It can be observed that there are numerous (ACFs) peaks but the largest peak of frequency 0.4880 at about $4.56 \times 10^{9}$ is the only value beyond the range of $4 \times 10^{9}$ as evident in Figure 1. Moreover, besides Periodogram that displays numerous peaks of greater and smaller values, the other ten major peak values ranging above $3 \times 10^{9}$ are tabulated in Table 4 , these peaks are also ranked as shown in column 6 of Table 2. Out of these next successive three highest major peaks are $3.72 \times 10^{9}, 3.64 \times 10^{9}$ and $3.58 \times 10^{9}$ at frequencies $0.1640,0.3840$ and 0.2880 , respectively. The fluctuating nature of Periodogram exhibits various moderate peaks at range of frequencies and lowest peak as well.

The Periodogram vs. periods of same set of observations I "Time of start of run since midnight" illustrated in Figure 3 manifests largest peaks of $4.56 \times 10^{9}$ at Period 2.0492. Periodogram having numerous peaks of larger and smaller values jumbled up within the period of 10 , whereas the range is 250 entries. Out of aforesaid major peaks, other ten values are tabulated in Table 4 indicates corresponding periods also next three successive highest major peaks are at periods of 6.0976, 2.6042 and 3.4722 , respectively.

The Tables 2, 3 and 4 exhibits a comparative study of the values obtained from Periodogram, ACF, PACF frequencies, autocorrelation and partial coefficient values for AR(1) models SNO $\mathrm{D}_{2} \mathrm{O}$ set of observations (Time of start of run since mid-night, in Sec), $\mathrm{N}=250$. Standard errors (SEs) are white noise estimates. Hamming weights ranges from 0.035714 to 0.446429 .

Figure 2 sketched the magnitude of Periodogram vs. frequency of our 2 nd set of observations, Time of start of run since midnight of SNO salt water sample taken containing 250 entries from 2 Nov 2001 to 08 Apr 2002. Five leading peak values are listed in Table 5. It can be observed that there are various (ACFs,) peaks but largest peak of frequency 0.1160 about $6.19 \times 10^{9}$ is one of the two values exceeding the range of $6 \times 10^{9}$ as represented in Figure 2. Furthermore, besides periodogram exhibits, numerous peaks of larger and smaller values, Seventeen major peaks values ranging above $3 \times 10^{9}$ are tabulated in Table 7 . These peaks are also ranked as shown in column 6 . Out of these three next major sequential higher peaks are $6.180 \times 10^{9}$, $5.324 \times 10$ and $5.12 \times 10^{9}$ at frequencies $0.320,0.3480$ and 0.1000 , respectively. The fluctuating nature of periodogram values exhibits various moderate and lowest peaks at the range of frequencies. 


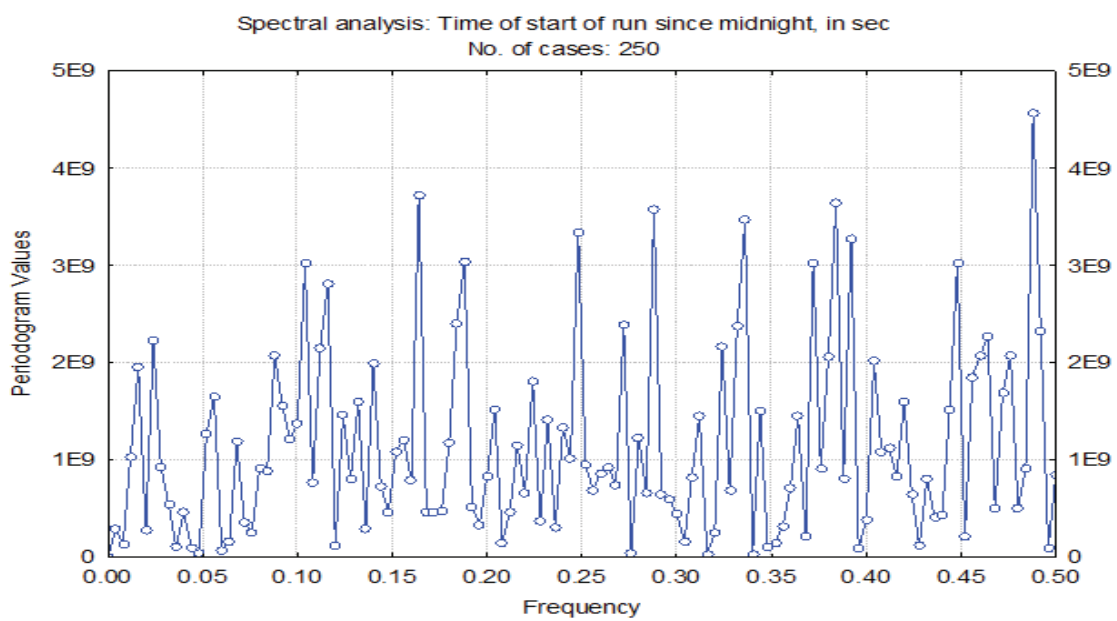

Fig. 1. Plot of Periodogram VS Frequency of Time of start of run since midnight of the neutrino interaction at SNO for heavy water set of observations

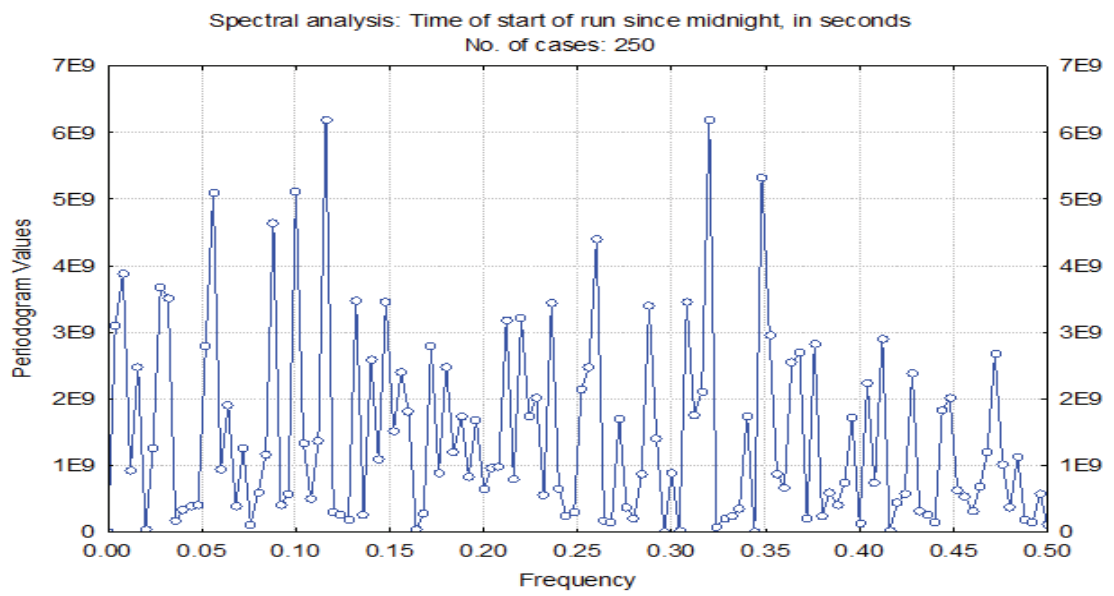

Fig. 2. Depiction of Periodogram VS Frequency of time of start of run since midnight of the neutrino interaction at SNO salt water set of observations

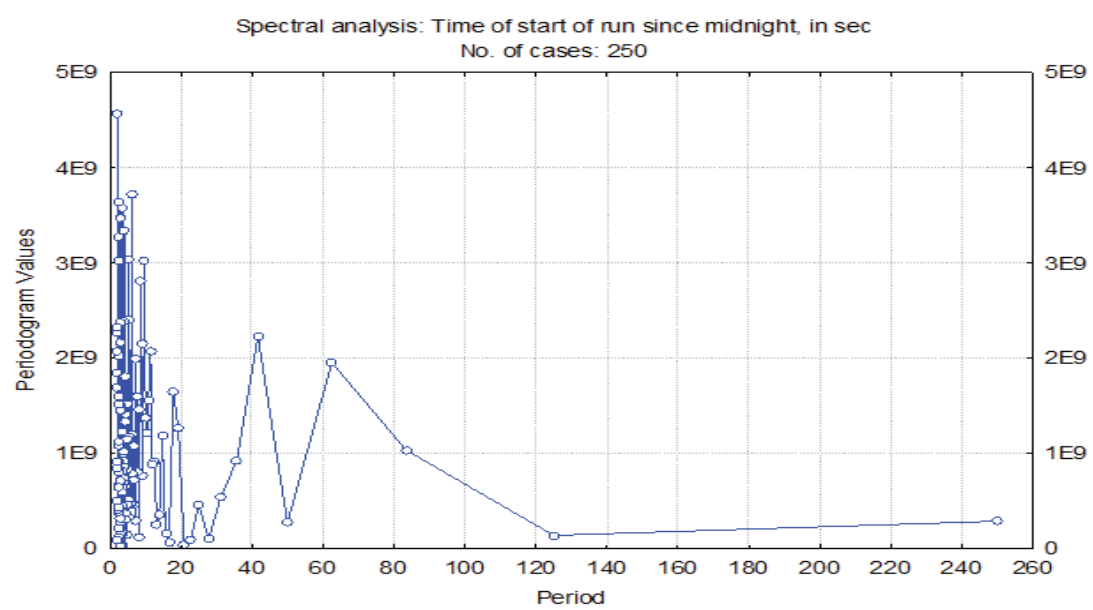

Fig. 3. Temporal behaviour of Periodogram for the time of start of run since midnight of the neutrino interaction at $\mathrm{SNO}_{2} \mathrm{O}$ set of observations 
The Periodogram against periods of said set of observations II exemplified in Fig. 4 indicates largest peaks of $6.19 \times 10$ at period 8.6207. Periodogram having numerous peaks of larger and smaller values jumbled up within the period of almost 10 values at the range of 250.Out of the seventeen aforesaid major peaks values tabulated in Table 7, next highest three major peaks are at periods $3.1250,2.8736$ and 10.0000 , respectively.

Tables 5, 6 and 7 exhibits a comparative study of the values obtained from Periodogram, ACF, PACF frequencies, autocorrelation and partial coefficient values for AR(1) models SNO Salt water set of observations (Time of start of run since mid-night, in $\mathrm{Sec}$ ), $\mathrm{N}=250$. Standard errors (SEs) are white noise estimates. Hamming weights ranges same from 0.035714 to 0.446429 .

The periodogram peaks against periods exemplified in Figures 3 and 4 are observed to be cluttered within the period of almost 10 values at the range of 250. The Periodogram of SNO heavy water $\left(\mathrm{D}_{2} \mathrm{O}\right)$ and salt water are also plotted against $\log$ period to discriminate the neat Periodogram as shown in Figures 5 and 6 . Thus we consider AR (1) to be the most appropriate model to study the SNO set of observations sample. However, for real representation, our model has rather temporal and spatial limitations in terms of stationarity, seasonality and periodical cycles[41].

\subsection{Spectral Density Contributions of Time of start of run (Neutrino interaction) since midnight of SNO set of observations using $D_{2} O$ and salt water}

The computed spectral density has been illustrated in Figure 7 that gives the runs in our $1^{\text {st }}$ set of observations; heavy water $\mathrm{D}_{2} \mathrm{O}$ sample outlined the magnitude of Spectral Density vs. frequency. Five Spectral Density peak values of Time of start of run since midnight of 1 stset of observations sample, comprising 250 entries from $2^{\text {nd }}$ Nov 1999 to $24^{\text {th }}$ Jun 2000 are presented in the Table8. There are numerous Spectral Density peaks but the biggest peak of frequency 0.4880 at about $2.8378 \times 10^{9}$ is the only value exceeding the range of $2.5 \times 10^{9}$ as exemplified in Figure 7. Furthermore, the Spectral Density exhibits numerous larger and smaller peak values, six major peaks values ranges above
$2 \times 10^{9}$ among total major eleven values, other five values are less than $2 \times 10^{9}$ as tabulated in Table 9, and the spectral density peaks are also ranked as shown in column 6 . Out of these three next highest major peaks are $2.46279 \times 10^{9}, 2.20599 \times 10^{9}$ and $2.11145 \times 10^{9}$ at frequencies $0.3840,0.3360$ and 0.1880 , respectively. The fluctuating behaviour of Spectral Density exhibits various moderate peaks at the range of frequencies and lowest peak as well.

The graph of Spectral Density against periods exemplified in Figure 9 does indicate the largest peaks of $2.8378 \times 10^{9}$ at Period 2.0492. The subjective graph having numerous peaks of larger and smaller values cluttered within the period of 10 values at the range of 250 Out of aforesaid major peaks, eleven values are tabulated in Table 9, three next successive higher major peaks are at periods of $2.6042,2.9762$ and 5.3191 respectively.

Figure 8 indicates Spectral Density peak values of Time of start of run since midnight of SNO salt water set of observations II. This sample has been taken comprising 250 entries from 2nd Nov 2001 to $8^{\text {th }}$ April 2002. In this illustration, five largest peak values are shown in the Table10. There are copious Spectral Density peaks but largest peak of frequency 0.3480 about $3.3548 \times 10^{9}$ is one of the four values exceeding the range of $3 \times 10^{9}$ as depicted in the Figure 8. Moreover, besides numerous Spectral Density peaks of larger and smaller values exhibited in above mentioned graph, fourteen major peaks values ranging above $2 \times 10^{9}$ are tabulated in Table 11, out of these three next higher major peaks are $3.2568 \times 10^{9}, 3.1921 \times 10^{9}$ and $3.1838 \times 10^{9}$ at frequencies $0.0560,0.1160$ and 0.3480 respectively. The changeable nature of Spectral Density exhibits various moderate and lowest peaks at a different range of frequencies.

In the Figure 10 Spectral Density has been plotted against time periods that examines largest peaks of $3.3548 \times 109$ at period 3.1250. Spectral Density having variable peaks of larger and smaller values disorderly manifested within the period of almost 10 values at the range of 250 . Out of the seventeen aforesaid major peaks values tabulated in Table 11, three next higher major peaks of values $3.2568 \times 10^{9}, 3.1921 \times 10^{9}$ and $3.1838 \times 10^{9}$ are at periods of $17.8571 \mathrm{~s}, 8.6207 \mathrm{~s}$ and $2.8736 \mathrm{~s}$ respectively. The Spectral Density peaks against 
Table 3. Spectral analysis: Time of start of run since midnight, in sec (No. of cases): 250 of $\mathrm{SNO}_{2} \mathrm{O}$ set of observations

\begin{tabular}{lccccc}
\hline S. No. & Frequency & Period & Periodogram & Density & Hamming Weight \\
\hline 1. & 0.000000 & & $2.778268 \mathrm{E}-22$ & $1.485993 \mathrm{E}+08$ & 0.035714 \\
2. & 0.004000 & 250.0000 & $2.877817 \mathrm{E}+08$ & $2.089476 \mathrm{E}+08$ & 0.241071 \\
3. & 0.008000 & 125.0000 & $1.378637 \mathrm{E}+08$ & $4.501975 \mathrm{E}+08$ & 0.446429 \\
4. & 0.012000 & 83.3333 & $1.034901 \mathrm{E}+09$ & $9.863664 \mathrm{E}+08$ & 0.241071 \\
5. & 0.016000 & 62.5000 & $1.954129 \mathrm{E}+09$ & $1.272145 \mathrm{E}+09$ & 0.035714 \\
\hline
\end{tabular}

Table 4. Spectral analysis: Time of start of run since midnight, in sec (No. of cases): 250 of $\mathrm{SNO} \mathrm{D}_{2} \mathrm{O}$ set of observations

\begin{tabular}{cccccc}
\hline S. No. & Frequency & Period & Periodogram & Density & Rank- Periodogram \\
\hline 1. & 0.104000 & 9.6154 & $3.022999 \mathrm{E}+09$ & $1.984294 \mathrm{E}+09$ & 10 \\
2. & 0.164000 & 6.0976 & $3.718237 \mathrm{E}+09$ & $2.023022 \mathrm{E}+09$ & 2 \\
3. & 0.188000 & 5.3191 & $3.034398 \mathrm{E}+09$ & $2.111449 \mathrm{E}+09$ & 8 \\
4. & 0.248000 & 4.0323 & $3.339811 \mathrm{E}+09$ & $2.035557 \mathrm{E}+09$ & 6 \\
5. & 0.288000 & 3.4722 & $3.578808 \mathrm{E}+09$ & $1.976691 \mathrm{E}+09$ & 4 \\
6. & 0.336000 & 2.9762 & $3.466150 \mathrm{E}+09$ & $2.205990 \mathrm{E}+09$ & 5 \\
7. & 0.372000 & 2.6882 & $3.022040 \mathrm{E}+09$ & $1.744537 \mathrm{E}+09$ & 11 \\
8. & 0.384000 & 2.6042 & $3.638505 \mathrm{E}+09$ & $2.462797 \mathrm{E}+09$ & 3 \\
9. & 0.392000 & 2.5510 & $3.272715 \mathrm{E}+09$ & $1.819047 \mathrm{E}+09$ & 7 \\
10. & 0.448000 & 2.2321 & $3.025479 \mathrm{E}+09$ & $1.850139 \mathrm{E}+09$ & 9 \\
11. & 0.488000 & 2.0492 & $4.564579 \mathrm{E}+09$ & $2.837839 \mathrm{E}+09$ & 1 \\
\hline
\end{tabular}

Table 5. Largest periodogram peaks of Time of start of run since midnight SNO salt water set of observations

\begin{tabular}{lccccc}
\hline Variable & \multicolumn{5}{c}{ Run start time since Midnight Five largest value } \\
\cline { 2 - 6 } & $(1)$ & $(2)$ & $(3)$ & $(4)$ & $(5)$ \\
\hline Periodogram peaks & $6.19 \times 10^{9}$ & $6.18 \times 10^{9}$ & $5.32 \times 10^{9}$ & $5.12 \times 10^{9}$ & $5.10 \times 10^{9}$ \\
Frequency & 0.1160 & 0.3200 & 0.3480 & 0.1000 & 0.0560 \\
Period & 8.6207 & 3.1250 & 2.8736 & 10.0000 & 17.8571 \\
\hline
\end{tabular}

Table 6. Spectral analysis: Time of start of run since midnight, in sec No. of cases: 250 Salt water set of observations

\begin{tabular}{cccccc}
\hline S. No. & Frequency & Period & Periodogram & Density & Hamming Weight \\
\hline 1. & 0.000000 & & $1.796133 \mathrm{E}-21$ & $1.776529 \mathrm{E}+09$ & 0.035714 \\
2. & 0.004000 & 250.0000 & $3.109118 \mathrm{E}+09$ & $2.468512 \mathrm{E}+09$ & 0.241071 \\
3. & 0.008000 & 125.0000 & $3.884858 \mathrm{E}+09$ & $2.794541 \mathrm{E}+09$ & 0.446429 \\
4. & 0.012000 & 83.3333 & $9.224573 \mathrm{E}+08$ & $2.056826 \mathrm{E}+09$ & 0.241071 \\
5. & 0.016000 & 62.5000 & $2.473276 \mathrm{E}+09$ & $1.518371 \mathrm{E}+09$ & 0.035714 \\
\hline
\end{tabular}

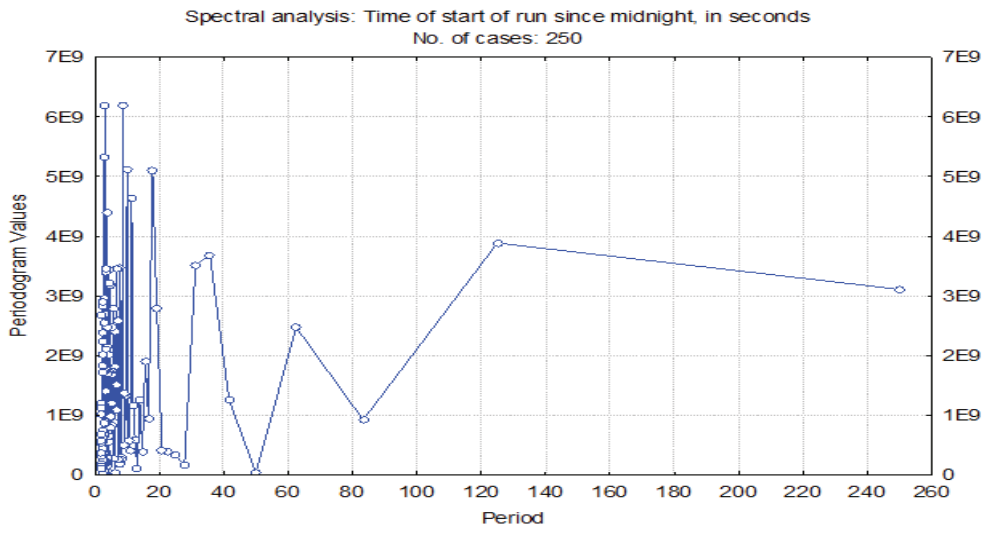

Fig. 4. Manifestation of Periodogram Vs Time Period of the time of start of run since midnight of the neutrino interaction at SNO Salt water set of observations 


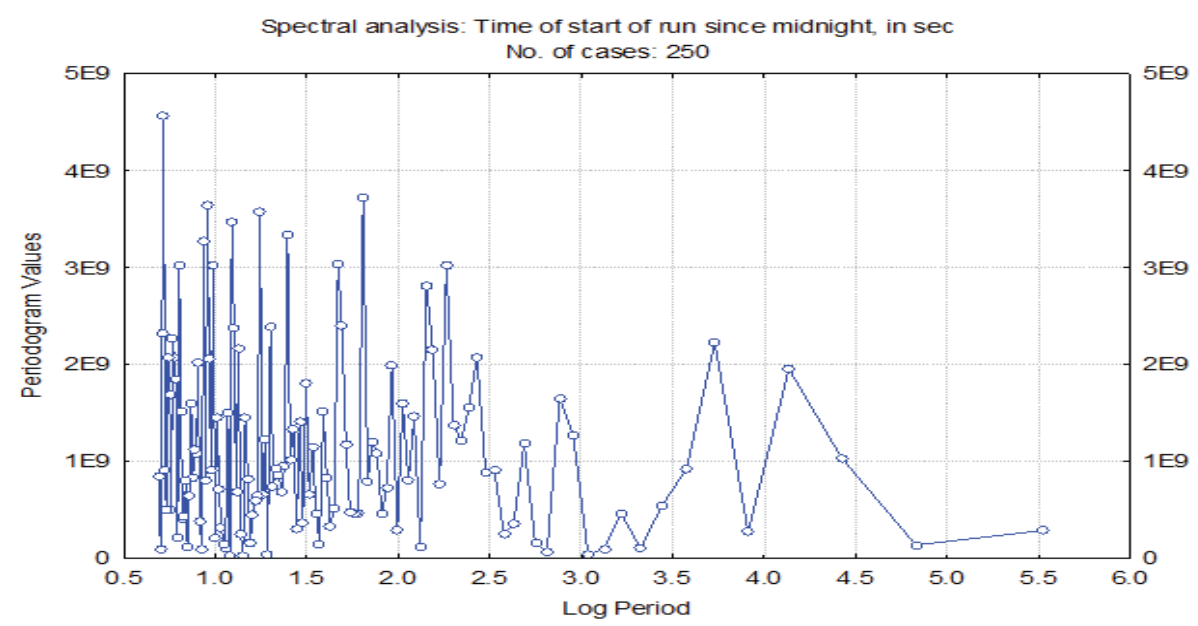

Fig. 5. Log period Vs Periodogram illustration of the time of start of run since midnight of the neutrino interaction at $\mathrm{SNO} \mathrm{D}_{2} \mathrm{O}$ set of observations.

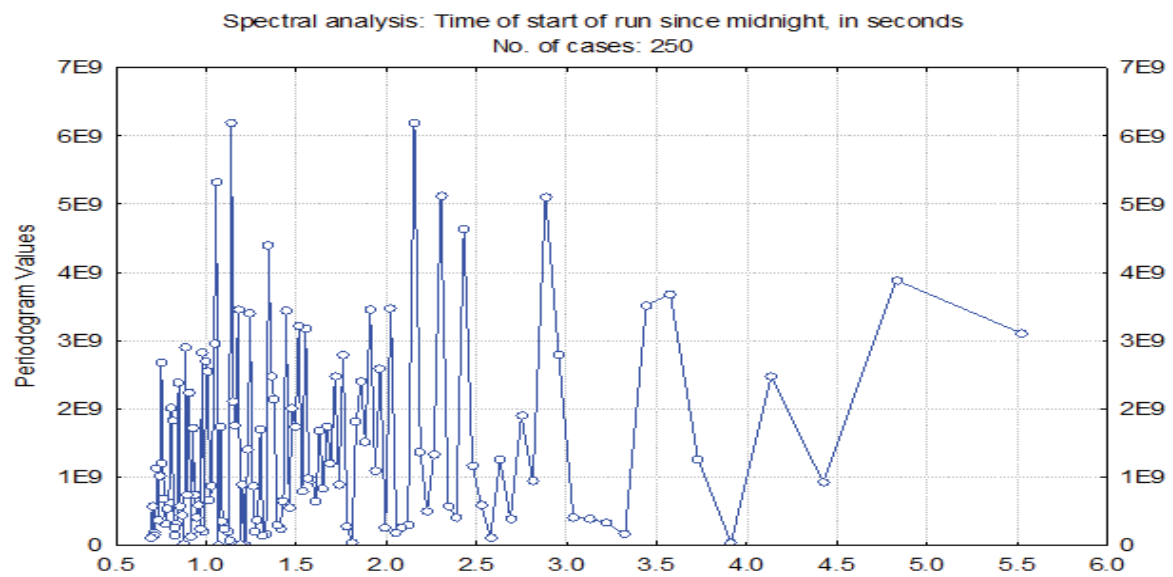

Fig. 6. Inspection of Periodogram versus Log period of time of start of run since midnight of the neutrino interaction at SNO Salt water set of observations

Table 7. Spectral analysis: Time of start of run since midnight, in sec No. of cases: 250 Salt water set of observations

\begin{tabular}{lccccc}
\hline S. No. & Frequency & Period & Periodogram & Density & Rank- Periodogram \\
\hline 1. & 0.008000 & 125.0000 & $3.884858 \mathrm{E}+09$ & $2.794541 \mathrm{E}+09$ & 8 \\
2. & 0.028000 & 35.7143 & $3.684055 \mathrm{E}+09$ & $2.802194 \mathrm{E}+09$ & 9 \\
3. & 0.032000 & 31.2500 & $3.513510 \mathrm{E}+09$ & $2.553848 \mathrm{E}+09$ & 10 \\
4. & 0.056000 & 17.8571 & $5.098201 \mathrm{E}+09$ & $3.256830 \mathrm{E}+09$ & 5 \\
5. & 0.088000 & 11.3636 & $4.629769 \mathrm{E}+09$ & $2.487906 \mathrm{E}+09$ & 6 \\
6. & 0.100000 & 10.0000 & $5.122073 \mathrm{E}+09$ & $2.778547 \mathrm{E}+09$ & 4 \\
7. & 0.116000 & 8.6207 & $6.186497 \mathrm{E}+09$ & $3.192087 \mathrm{E}+09$ & 1 \\
8. & 0.132000 & 7.5758 & $3.471098 \mathrm{E}+09$ & $1.757887 \mathrm{E}+09$ & 11 \\
9. & 0.148000 & 6.7568 & $3.462765 \mathrm{E}+09$ & $2.351670 \mathrm{E}+09$ & 12 \\
10. & 0.212000 & 4.7170 & $3.185960 \mathrm{E}+09$ & $1.998613 \mathrm{E}+09$ & 17 \\
11. & 0.220000 & 4.5455 & $3.214549 \mathrm{E}+09$ & $2.231455 \mathrm{E}+09$ & 16 \\
12. & 0.236000 & 4.2373 & $3.432427 \mathrm{E}+09$ & $1.903511 \mathrm{E}+09$ & 14 \\
13. & 0.260000 & 3.8462 & $4.395321 \mathrm{E}+09$ & $2.682679 \mathrm{E}+09$ & 7 \\
14. & 0.288000 & 3.4722 & $3.392228 \mathrm{E}+09$ & $2.071161 \mathrm{E}+09$ & 15 \\
15. & 0.308000 & 3.2468 & $3.456059 \mathrm{E}+09$ & $2.077655 \mathrm{E}+09$ & 13 \\
16. & 0.320000 & 3.1250 & $6.180317 \mathrm{E}+09$ & $3.354829 \mathrm{E}+09$ & 2 \\
17. & 0.348000 & 2.8736 & $5.324433 \mathrm{E}+09$ & $3.183849 \mathrm{E}+09$ & 3 \\
\hline
\end{tabular}


Table 8. Largest Spectral density peaks of Time of start of run since midnight of SNO $\mathrm{D}_{2} \mathrm{O}$ set of observations

\begin{tabular}{lccccc}
\hline Variable & \multicolumn{4}{c}{ Run start time since Midnight: Five largest values } \\
\cline { 2 - 6 } & $(1)$ & $(2)$ & $(3)$ & $(4)$ & $(5)$ \\
\hline Spectral density peaks & $2.8378 \times 10^{9}$ & $2.4628 \times 10^{9}$ & $2.2060 \times 10^{9}$ & $2.1115 \times 10^{9}$ & $2.0356 \times 10^{9}$ \\
Frequency & 0.4880 & 0.3840 & 0.3360 & 0.1880 & 0.2480 \\
Period & 2.0492 & 2.6042 & 2.9762 & 5.3191 & 4.0323 \\
\hline
\end{tabular}

Table 9. Spectral analysis: Time of start of run since midnight, in sec (No. of cases): 250 of SNO D2O set of observations

\begin{tabular}{cccccc}
\hline S. No. & Frequency & Period & Periodogram & Density & Rank- Spec Density \\
\hline 1. & 0.104000 & 9.6154 & $3.022999 \mathrm{E}+09$ & $1.984294 \mathrm{E}+09$ & 7 \\
2. & 0.164000 & 6.0976 & $3.718237 \mathrm{E}+09$ & $2.023022 \mathrm{E}+09$ & 6 \\
3. & 0.188000 & 5.3191 & $3.034398 \mathrm{E}+09$ & $2.111449 \mathrm{E}+09$ & 4 \\
4. & 0.248000 & 4.0323 & $3.339811 \mathrm{E}+09$ & $2.035557 \mathrm{E}+09$ & 5 \\
5. & 0.288000 & 3.4722 & $3.578808 \mathrm{E}+09$ & $1.976691 \mathrm{E}+09$ & 8 \\
6. & 0.336000 & 2.9762 & $3.466150 \mathrm{E}+09$ & $2.205990 \mathrm{E}+09$ & 3 \\
7. & 0.372000 & 2.6882 & $3.022040 \mathrm{E}+09$ & $1.744537 \mathrm{E}+09$ & 11 \\
8. & 0.384000 & 2.6042 & $3.638505 \mathrm{E}+09$ & $2.462797 \mathrm{E}+09$ & 2 \\
9. & 0.392000 & 2.5510 & $3.272715 \mathrm{E}+09$ & $1.819047 \mathrm{E}+09$ & 10 \\
10. & 0.448000 & 2.2321 & $3.025479 \mathrm{E}+09$ & $1.850139 \mathrm{E}+09$ & 9 \\
11. & 0.488000 & 2.0492 & $4.564579 \mathrm{E}+09$ & $2.837839 \mathrm{E}+09$ & 1 \\
\hline
\end{tabular}

Table 10. Largest Spectral Density peaks of Time of start of run since midnight SNO salt water set of observations

\begin{tabular}{llllll}
\hline Variable & \multicolumn{5}{c}{ Run start time since Mid night Five largest value } \\
\cline { 2 - 6 } & \multicolumn{1}{c}{$(1)$} & \multicolumn{1}{c}{$(2)$} & \multicolumn{1}{c}{$(3)$} & \multicolumn{1}{c}{$(4)$} & $(5)$ \\
\hline Spectral Density peaks & $3.3548 \times 10^{9}$ & $3.2568 \times 10^{9}$ & $3.1921 \times 10^{9}$ & $3.1838 \times 10^{9}$ & $2.8022 \times 10^{9}$ \\
Frequency & 0.3480 & 0.0560 & 0.1160 & 0.3480 & 0.0280 \\
Period & 3.1250 & 17.8571 & 8.6207 & 2.8736 & 35.7143 \\
\hline
\end{tabular}

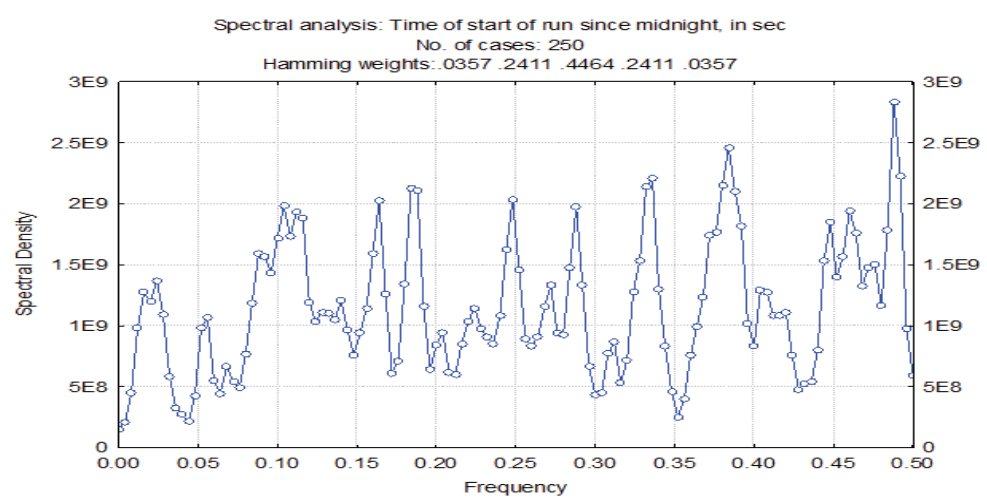

Fig. 7. Spectral Density versus Frequency of time of start of run since midnight of the neutrino interaction at $\mathrm{SNO}_{2} \mathrm{O}$ set of observations.

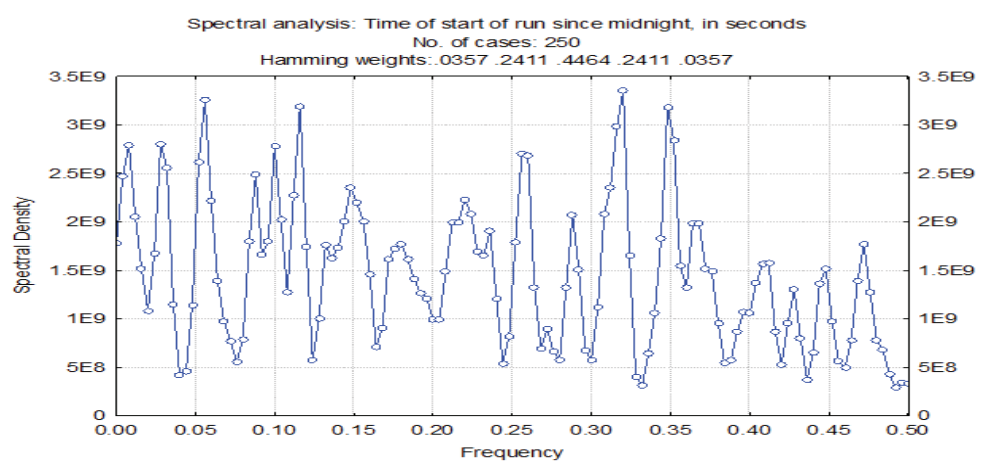

Fig. 8. Display of Spectral Density versus Frequency of time of start of run since midnight of the neutrino interaction at SNO Salt water set of observations 
periods displayed in Figures 9 and 10 illustrated to be cluttered within the period of almost 10 values at the range of 250. The Spectral Density of set of observation I, SNO heavy water $\left(\mathrm{D}_{2} \mathrm{O}\right)$ and set of observation II, salt water are also plotted against log period shown in Figures 11 and 12.

\section{ANALYSIS}

While analyzing the graphs and values, it has been concluded that comparatively, the Time of start of run since midnight of SNO salt water set of observations has highest periodogram peaks of

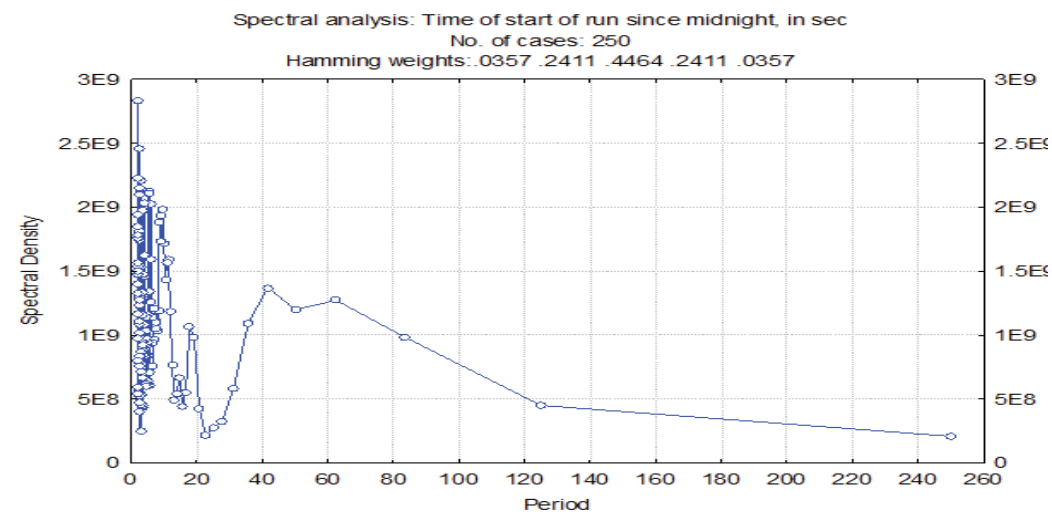

Fig. 9. Depiction of Spectral Density versus Period of time of start of run since midnight of the neutrino interaction at $\mathrm{SNO}_{2} \mathrm{O}$ set of observations.

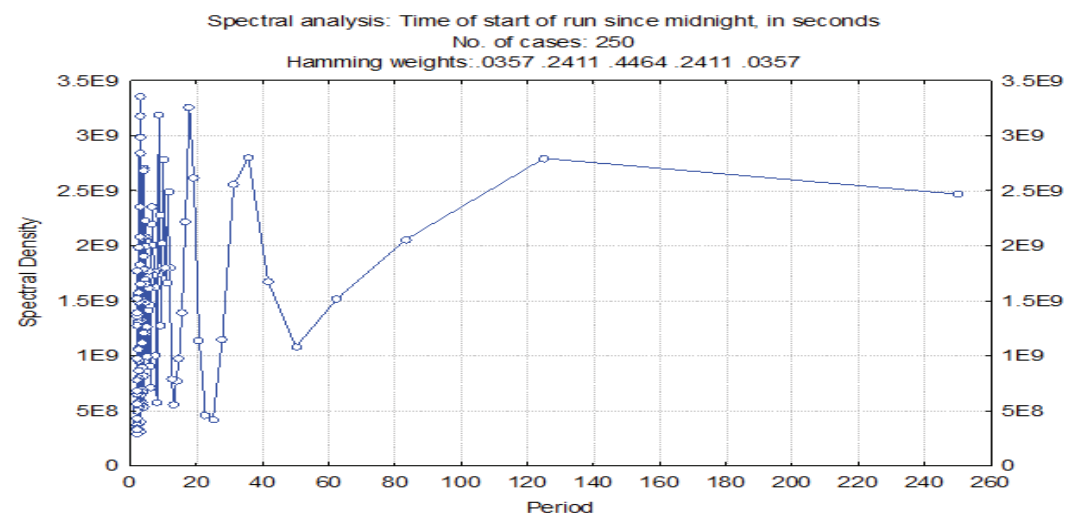

Fig. 10. Illustration of Spectral Density versus Period of time of start of run since midnight of the neutrino interaction at SNO Salt water set of observations

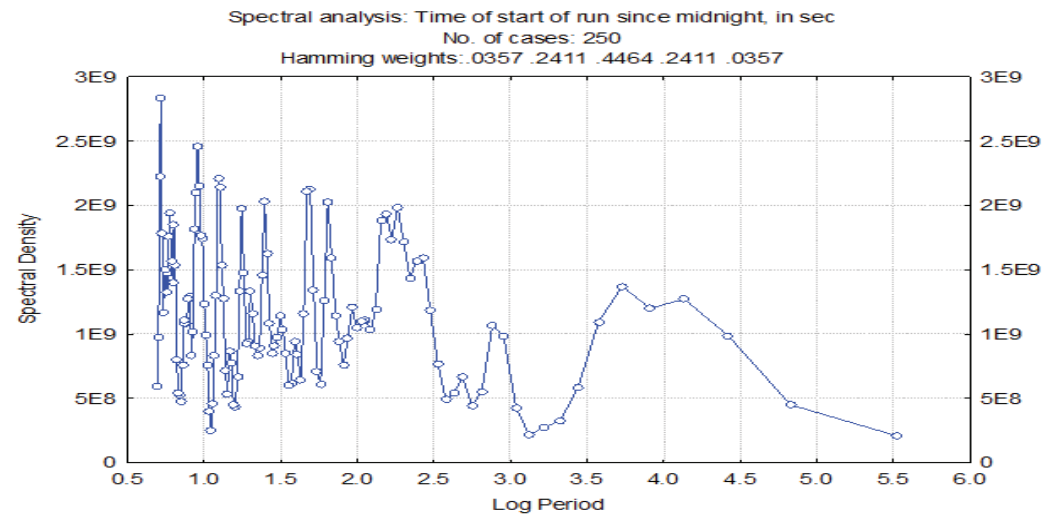

Fig. 11. Display of Spectral Density versus Log Period of time of start of run since midnight of the neutrino interaction at $\mathrm{SNO}_{2} \mathrm{O}$ set of observations. 


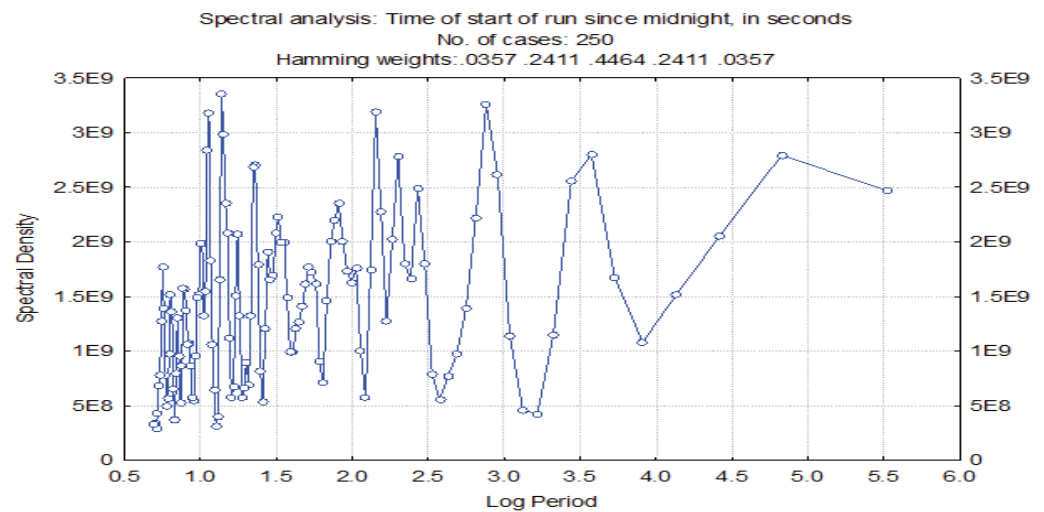

Fig. 12. Shows the Spectral Density versus Log Period behavior of time of start of run since midnight of the neutrino interaction at SNO Salt water set of observation

Table 11. Spectral analysis: Time of start of run since midnight, in sec No. of cases: 250 Salt water set of observations

\begin{tabular}{lccccc}
\hline S. No. & Frequency & Period & Periodogram & Density & Rank- Spec Density \\
\hline 1. & 0.008000 & 125.0000 & $3.884858 \mathrm{E}+09$ & $2.794541 \mathrm{E}+09$ & 6 \\
2. & 0.028000 & 35.7143 & $3.684055 \mathrm{E}+09$ & $2.802194 \mathrm{E}+09$ & 5 \\
3. & 0.032000 & 31.2500 & $3.513510 \mathrm{E}+09$ & $2.553848 \mathrm{E}+09$ & 9 \\
4. & 0.056000 & 17.8571 & $5.098201 \mathrm{E}+09$ & $3.256830 \mathrm{E}+09$ & 2 \\
5. & 0.088000 & 11.3636 & $4.629769 \mathrm{E}+09$ & $2.487906 \mathrm{E}+09$ & 10 \\
6. & 0.100000 & 10.0000 & $5.122073 \mathrm{E}+09$ & $2.778547 \mathrm{E}+09$ & 7 \\
7. & 0.116000 & 8.6207 & $6.186497 \mathrm{E}+09$ & $3.192087 \mathrm{E}+09$ & 3 \\
8. & 0.132000 & 7.5758 & $3.471098 \mathrm{E}+09$ & $1.757887 \mathrm{E}+09$ & 17 \\
9. & 0.148000 & 6.7568 & $3.462765 \mathrm{E}+09$ & $2.351670 \mathrm{E}+09$ & 11 \\
10. & 0.212000 & 4.7170 & $3.185960 \mathrm{E}+09$ & $1.998613 \mathrm{E}+09$ & 15 \\
11. & 0.220000 & 4.5455 & $3.214549 \mathrm{E}+09$ & $2.231455 \mathrm{E}+09$ & 12 \\
12. & 0.236000 & 4.2373 & $3.432427 \mathrm{E}+09$ & $1.903511 \mathrm{E}+09$ & 16 \\
13. & 0.260000 & 3.8462 & $4.395321 \mathrm{E}+09$ & $2.682679 \mathrm{E}+09$ & 8 \\
14. & 0.288000 & 3.4722 & $3.392228 \mathrm{E}+09$ & $2.071161 \mathrm{E}+09$ & 14 \\
15. & 0.308000 & 3.2468 & $3.456059 \mathrm{E}+09$ & $2.077655 \mathrm{E}+09$ & 13 \\
16. & 0.320000 & 3.1250 & $6.180317 \mathrm{E}+09$ & $3.354829 \mathrm{E}+09$ & 1 \\
17. & 0.348000 & 2.8736 & $5.324433 \mathrm{E}+09$ & $3.183849 \mathrm{E}+09$ & 4 \\
\hline
\end{tabular}

$6.91 \times 10^{9}$ for $\mathrm{SNO} \mathrm{D}_{2} \mathrm{O}$ set of observations having highest peak of $4.56 \times 10^{9}$. These depict the start of neutrino interaction has higher periodogram value in salt water set of observations. Similarly, the Time of start of run since midnight of SNO salt water set of observations posses highest Spectral density peaks of $3.3548 \times 10^{9}$ in comparison to $\mathrm{SNO} \mathrm{D}_{2} \mathrm{O}$ set of observations having highest peak of $2.8378 \times 10^{9}$ that depicts neutrino interaction has higher Spectral density value in salt water set of observations.

The variable analyzed in this study is "Time of start of run since midnight" means the value so recorded is the time in Sec I, start time of neutrino interaction. Higher peaks of Spectral density and Periodogram indicates delayed emission of neutrino interaction; the set of observations discussed in this study, are recorded at the same geographical position, in the same laboratory, in almost same environment, the time frame as mentioned above is carefully selected to be of the same date and month despite different years for better similarity. Besides the time frame, the difference is medium, i.e. heavy water $\left(\mathrm{D}_{2} \mathrm{O}\right)$ and Salt water. The observation not further deliberated here but will be taken up in the next such study.

Both set of SNO observations referred here have been recorded two variables, one analyzed in this piece of study. Analysis of the other variable, "length of run in Sec" will be dealt with in next analyses. Before finalizing the neutrino emissions and their capture status, we have considered referring some ultimate facts about the methodology adopted for 
analyses, i.e., Periodogram. It exhibits in a sample distribution having a relatively straightforward interpretation of Periodogram for which data exhibit strong cyclic variation at one of the Fourier components. In another sample distribution having a more stable pattern of the Periodogram, where the cyclic variation is weaker and not necessarily at frequencies corresponding to any of the Fourier components.

Finally, referring to a time series investigation it quotes that the emphasis has been put on the difficulties which can arise in the interpretation of Periodogram. In a sample distribution having a long upper tail, the effect of which is that the Periodogram includes many visually striking peaks despite the complete absence of any genuine cyclic effect" [37].

\section{CONCLUSION}

It has been established that neutrinos existence and emissions from deep space has been detected in world laboratories as mentioned in the above sections. These laboratories are recording regularly neutrinos set of observations to understand behavior and characteristic of the same. The samples of these observations recorded in the Sudbury Neutrino Observatory (SNO), Ontario, Canada, from Nov 1999 to May 2001 of heavy water $\left(\mathrm{D}_{2} \mathrm{O}\right)$ and setII from Jul 2001 to Aug 2003 of salt water with Run start Time since midnight are analyzed here to examine time based variations using standard software to construct periodogram and Spectral density curves that are exhibited both in time and frequency domains. No sign of time base variation has been manifested in both set of observations in joint analyses of the two sets of observations as it has been illustrated by One of SNO papers during the year 2005 [37].

\section{ACKNOWLEDGMENT}

I wish to endorse my sincere thanks to Canadian laboratory known as Sudbury Neutrino Observatory (SNO) for providing the set of observations to undertake this kind of research work in Pakistan, where there is no neutrino detection laboratory does exist. Moreover, I wish that I may be obliged to enjoy the support of the University of Karachi and the Institute of Space Science and Technology and guidance afforded by my Supervisor and colleagues during the course of this work. This research work is part of my $\mathrm{Ph}$. D dissertation.

\section{CONFLICT OF INTEREST}

On behalf of all authors, the corresponding author states that there is no conflict of interest.

\section{REFERENCES}

1. Winter, K. Neutrino Physics. Cambridge University Press (1991).

2. Pauli, W. letter of the $4^{\text {th }}$ of December 1930, Pauli Archive at CERN.

3. Zralek, M. 50 years of Neutrino Physics, arXiv:1012.2390v1 (2010).

4. Fritsch, U.: Cluster search for neutrinos from Active Galactic Nuclei (Doctoral dissertation, FriedrichAlexander-Universität Erlangen-Nürnberg, (2014).

5. Bahcall, J. An introduction to solar neutrino research. arXiv preprint hep-ph/9711358(1997).

6. Takita, M.: Frontiers of Neutrino Astrophysics, p. 147(1993).

7. Davis, R.: A review of the Homestake solar neutrino experiment. Progress in Particle and Nuclear Physics, 32, 13-32(1994).

8. Bahcall, J. N., Basu, S., \&Pinsonneault, M. H.: How uncertain are solar neutrino predictions? Physics Letters B,433(1-2), 1-8(1998).

9. Suzuki, Y.: Kamiokande solar neutrino results. Nuclear Physics B-Proceedings Supplements, 38(1-3), 54-59(1995).

10. Fukuda, Y., Hayakawa, T., Inoue, K., Ishihara, K., Ishino, H., Joukou, S., ...\& Matsumoto, K.: Solar neutrino data covering solar cycle 22. Physical Review Letters, 77(9), 1683(1996).

11. Anselmann, P., Fockenbrock, R., Hampel, W., Heusser, G., Kiko, J., Kirsten, T., ...\&Sann, M.: First results from the $51 \mathrm{Cr}$ neutrino source experiment with the GALLEX detector. Physics Letters B, 342(1-4), 440-450 (1995).

12. Abdurashitov, J. N., Faizov, E. L., Gavrin, V. N., Gusev, A. O., Kalikhov, A. V., Knodel, T. V., \&Shalagin, A. M.: Results from SAGE; The Russian-American gallium solar neutrino experiment. Physics Letters B, 328(1-2), 234-248 (1994).

13. LoSecco, J. M.: The History of "Anomalous" Atmospheric Neutrino Events: A First Person Account. Physics in Perspective, 18(2), 209241(2016).

14. Davis Jr, R., Harmer, D. S., \& Hoffman, K. C.: Search for neutrinos from the Sun. Physical Review 
Letters, 20(21), 1205 (1968).

15. Sheldon, W. R.: Possible relation of a null solar neutrino flux to the II year solar cycle. Nature, 221(5181), 650 (1969).

16. D'Alessi, L., Vecchio, A., Carbone, V., Laurenza, M., \&Storini, M.: Quasi-biennial modulation of the solar neutrino flux: a "Telescope" for the solar interior. Journal of Modern Physics, 4(04), 49(2013).

17. Pontieri, A., Lepreti, F., Sorriso-Valvo, L., Vecchio, A., \& Carbone, V.: A simple model for the solar cycle. Solar Physics, 213(1), 195-201(2003).

18. Sakurai, K.: Quasi-biennial variation of the solar neutrino flux and solar activity. Nature, 278(5700), 146 (1979).

19. Chen, M. C.: Solar neutrino experiments: Status and prospects. Physics of Particles and Nuclei, 42(4), 558(2011).

20. May-Britt K. Space Physics - An Introduction to Plasma and Particles in the Heliosphere and Magnetospheres, Springer-Verlag Berlin Heidelberg $3^{\text {rd }}$ Edition (2004).

21. Bradley W. Carroll, Dale A. Ostlie: An Introduction to Modern Astrophysics $2^{\text {nd }}$ Edition, Pearson International Edition (2007).

22. Ahmad et al.: Measurement of the charged current interactions produced by B-8 solar neutrinos at the Sudbury Neutrino Observatory. Phys. Rev. Lett, 87, 071301(2001).

23. Ahmad, Q. R., Allen, R. C., Andersen, T. C., Anglin, J. D., Barton, J. C., Beier, E. W., ...\&Blevis, I.: Direct evidence for neutrino flavor transformation from neutral-current interactions in the Sudbury Neutrino Observatory. Physical review letters, 89(1), 011301(2002).

24. SNO collaboration: Measurement of the total active 8B solar neutrino flux at the Sudbury Neutrino Observatory with enhanced neutral current sensitivity. arXiv preprint nucl-ex/0309004(2003).

25. Waller, D. Results from the Sudbury Neutrino Observatory, SLAC Summer Institute on Particle Physics (SSI04), 2-13, (2004).

26. Ahmad, Q. R., Allen, R. C., Andersen, T. C., Anglin, J. D., Barton, J. C., Beier, E. W., ... \&Blevis, I.: Measurement of day and night neutrino energy spectra at SNO and constraints on neutrino mixing parameters. Physical Review Letters, 89(1), 011302(2002).

27. Ahmad, Q. R., Allen, R. C., \& Andersen, T. C.: Measurement of the rate of $v e+d \rightarrow p+p+e^{-}$ interactions produced by $8 B$ solar neutrinos at the Sudbury Neutrino Observatory. Phys. Rev. Lett,
87(7), 071301(2001).

28. Ahmed, S. N., Anthony, A. E., Beier, E. W., Bellerive, A., Biller, S. D., Boger, J., ...\& Bullard, T. V.: Constraints on nucleon decay via invisible modes from the Sudbury Neutrino Observatory. Physical review letters, 92(10), 102004 (2004).

29. Ahmed, S., Anthony, A. E., Beier, E. W., Bellerive, A., Biller, S. D., Boger, J., ...\& Bullard, T. V.: Measurement of the Total Active B 8 Solar Neutrino Flux at the Sudbury Neutrino Observatory with Enhanced Neutral Current Sensitivity. Physical review letters, 92(18), 181301(2004).

30. Ewan, G. T., \& Davidson, W. F.: Early development of the underground SNO laboratory in Canada. Physics in Canada (2005).

31. Boger, J., Hahn, R. L., Rowley, J. K., Carter, A. L., Hollebone, B., Kessler, D., ... \& Grant, D. R.: The Sudbury neutrino observatory. Nuclear Instruments and Methods in Physics Research Section A: Accelerators, Spectrometers, Detectors and Associated Equipment, 449(1-2), 172-207(2000).

32. Makridakis S \& Steven C. W., Forecasting: methods and applications, John Wiley, New York (1985).

33. Montgomery D C and Johnson L A., Forecasting and time series analysis, McGrew-Hill, New York (1990).

34. Millard S. P., Environmental Stats for S-Plus, (Springer-Verlag), New York (1998).

35. Chatfield C., The analysis of Time Series: an introduction, 4/e, Chapman \& Hall, London (1989).

36. Pandit S M and Wu S-M., Time Series and System Analysis with applications, New York: J Wiley (1985).

37. Diggle, P. J.: Time series; a bio-statistical introduction (No. 04; QA280, D5.) (1990).

38. SNO Collaboration: A Search for Periodicities in the 8B Solar Neutrino Flux Measured by the Sudbury Neutrino Observatory (2005).

39. Bahcall, J. N.: Solar neutrinos. i. theoretical. Physical Review Letters, 12(11), 300(1964).

40. Collaboration, S. N. O.: HOWTO use the SNO Salt flux results (2003).

41. Tong, H.: Non-linear time series: a dynamical system approach. Oxford University Press(1990).

42. Mc Taggart, R. (2017). Simulation of Neutrino Detection and Background Rejection for a Heavy Liquid Scintillator in a Space Environment. Marshall Space Flight Center Faculty Fellowship Program, 102.

43. Davis Jr, R.: Solar neutrinos. ii. experimental. Physical Review Letters, 12(11), 303(1964). 
